\title{
The Superior Perspective for Management Thinking
}

Complex management issues require a switch in perspective from reductionist to holistic thinking

\author{
Uyiosa Omoregie
}

April 2017

uyiosaomoregie@yahoo.co.uk

\begin{abstract}
Two types of thinking/perspectives are contrasted: reductionist and holistic. The superior of the two is highlighted by its effectiveness in the management of complex phenomena ranging from business management to social policy. Reductionist thinking has its benefits when dealing with simpler phenomena but has severe limitations and can be a blind-spot for managers. As the world we live in now is characterized by increasing complexity, managers must switch from reductionist to holistic thinking in order to make the right interventions in complex issues.
\end{abstract}

Key words: Holistic, Reductionist, Complexity, Management 


\section{Introduction}

Is there a superior way for managers to think? Here, 'managers' defined in the broadest of terms to mean anyone involved in the process of realisation of objectives and goals defined by humans. This follows the definition of 'management' by Petersen and Plowman [1]:

Management may be defined as the process by means of which the purpose and objectives of a particular human group are determined, clarified and effectuated.

Scholars and commentators have repeatedly pointed out that we live in a period of rapid change and overwhelming complexity[2]-[5]. It has been suggested that old ways of thinking will be incapable of managing the complexity of modern life [6][2][7][8][4]. Mishra [9] argues that mankind "cannot understand this crisis because our dominant intellectual concepts and categories seem unable to process an explosion of uncontrolled forces." Modern social systems have been described as possessing counterintuitive behaviour[7[, and society "becomes frustrated as repeated attacks on deficiencies in social systems lead only to worse symptoms." Some of the urgent challenges facing mankind include climate change, global terrorism, biodiversity depletion, global financial crises, transnational money laundering, global economic inequality, water shortage, corruption, global disease epidemics and xenophobia.

In 1918, the American intellectual Henry Adams [10] suggested that "a new social mind" is required by mankind. This new social mind must replace the old mind, if mankind will competently manage modern life's complexity. Adams' thoughts were in response to the changes imposed on mankind by the industrial age. Almost 100 years later, in 2017, another American social commentator, Mark Zuckerberg [11], in a 6,000-word manifesto called for the creation of "a new social infrastructure" to enable humanity "take the next step." The move to create a new social mind and form effective social infrastructure will require a different mode of thinking, a different perspective. The predominant mode of thinking or perspective, for management - used to determine, clarify and effectuate human purposes and objectives - is reductionist/linear thinking. The reductionist/linear perspective has been very effective and successful, to a large extent, 
especially in the world of science. In contrast to reductionist/linear thinking and perspective is the holistic/complexity thinking and perspective.

This paper highlights the difference between reductionist/linear and holistic/complexity thinking. Deeper aspects of the contrast between the two perspectives and thought systems are presented. The geographic prevalence of these systems of thought as well as the ideological contrasts are discussed. Next, holistic/complexity thinking is presented as the superior perspective for managers. Examples of different fields of application are used to illustrate the effectiveness of the holistic/complexity perspective for management of issues.

\section{Origins of two systems of thinking}

Daniel Kahneman was awarded the Nobel Prize for Economics[12](officially The Sveriges Riksbank Prize in Economic Sciences in Memory of Alfred Nobel, 2002), for "having integrated insights from psychological research into economic science, especially concerning human judgment and decision-making under uncertainty." Kahneman collaborated with Amos Tversky for most of his career, together they revealed systematic errors in human thinking, linked to cognition rather than emotions. Kahenman[13] went on to describe two systems of thought: System 1 'fast' and System 2 'slow'. System 1 is reflexive and quick, relatively effortless with "no sense of voluntary control." System 2 is more effortful and mentally tasking, more voluntary, typically used for complex calculations: "the operations of System 2 are often associated with the subjective experience of agency, choice and concentration."

The two modes of thought/perspective presented in this paper (reductionist/linear and holistic/complexity) belong to Kahnemen's System 2: they are deliberate, conscious, voluntary and typically used by managers. The role that geography and culture play to shape modes of thinking and perspectives, has been generally underestimated. Robson [14] went as far to say that the farming habits of our ancestors (the kind of crops they cultivated) and even physical boundaries, such as the presence of a river, may demarcate two different thinking styles. Henrich et al.[15]show that the reductionist/linear "analytic" perspective is valued more in Western culture. In East Asian culture, the holistic/complexity perspective is more valued. Henrich et al.[15] further highlighted findings from research showing that 
Western people tend to use a decontextualized perspective when explaining behaviour.

Henrich et al.[15]define "analytic" and "holistic" thought thus:

Holistic thought involves an orientation to the context or field as a whole, including attention to relationships between a focal object and the field, and a preference for explaining and predicting events on the basis of such relationships. Analytic thought involves a detachment of objects from contexts, a tendency to focus on objects' attributes, and a preference for using categorical rules to explain and predict behavior. This distinction between habits of thought rests on a theoretical partition between two reasoning systems. One system is associative, and its computations reflect similarity and contiguity (i.e., whether two stimuli share perceptual resemblances and cooccur in time); the other system relies on abstract, symbolic representational systems, and its computations reflect a rulebased structure...

Difference in the prevalence of each mode of thought in the West and the East, is also linked to the self-views of the people. Westerners tend to have an independent self-view while East Asians tend towards an interdependent self-view, these two self-views are connected to the individualistic or collectivist construct[16][17]. East Asians tend to have little or no self-serving biases, they may even have self-effacing biases instead[18]. Cross[19] showed that a sample of American professors had incredible self-serving biases: 94 percent of them rated themselves better than their peers. However, as both the "analytic"/reductionist and Holistic/complexity cognitive systems are System 2 'slow-thinking' constructs, they are available to all human adults. These two systems of thought are not reflexive or automatic but learned and hence can be put to use deliberately, by any manager. The cultural setting may tend to influence the prevalence of one mode of thinking over the other.

\subsection{Reductionist/complexity/linear/'analytical' thinking}

'Reductionism' in simplest terms means the breaking down (reduction) of complicated phenomena into smaller parts, for easier understanding. This attempt to explain entire systems in terms of their individual, constituent parts and their interactions, has been successfully applied in the sciences: 'methodological reductionism.' In reductionist methodology, 'cause' and 'effect' are usually not far from each other. The reductionist perspective often reveals linear causality. The Enlightenment philosopher René Descartes 
greatly influenced the development of the reductionist method. Descartes[20] worked to differentiate superstitious beliefs from the scientific method. Descartes believed that only science/philosophy could provide truth superstition could not. This Cartesian method (which involved decomposing the problem into smaller aspects for individual scrutiny, then, if necessary, reconstructing the decomposed parts into a whole again, to understand the integrated form) would prove crucial to the understanding of science during and after the Enlightenment period. He summarized his method in four precepts, the second and the third precepts described the need for analytic reduction to "...divide each of the difficulties under examination into as many parts as possible, and as might be necessary for its adequate solution...[then] commencing with objects the easiest to know, I might ascend by little and little, and, as it were, step by step to the knowledge of the more complex; assigning in thought a certain order even to those objects which in their own nature do not stand in relation of antecedence and sequence." Apart from Isaac Newton, probably no other person has exerted as much influence over scientific/reductionist thinking as did René Descartes. Newton proved the world to be a mechanical and deterministic universe. The reductionistmechanical worldview of Descartes and Newton has reigned supreme especially in the sciences. But, as Wolfram[21] states, this thinking/perspective has its shortcomings:

In the existing sciences much of the emphasis over the past century or so has been on breaking systems down to find their underlying parts, then trying to analyze these parts in as much detail as possible. And particularly in physics this approach has been sufficiently successful that the basic components of everyday systems are now completely known. But just how these components act together to produce even some of the most obvious features of the overall behavior we see has in the past remained an almost complete mystery.

\subsection{Holistic/complexity/non-linear/'systems' thinking}

Before the Descartes-Newton perspective came to dominate Western thinking, the worldview of the ancient Greek philosopher and scientist Aristotle, reigned supreme. Arististole's aim was to catalogue the world and organize it by observing patterns and systems in all complexity. Aristotle believed that the whole is something distinct from the parts it is composed of - the total of the constituent parts is not just made up of a heap of the parts. He believed that the study of the unity of such complexes is distinct from the 
study of the parts. True knowledge for Aristotle, comes not necessarily from breaking up the parts, but from the consideration of the whole rather than the constituent parts[22].

'Complexity' was "roughly" defined by Simon[23] as "a system made up of a large number of parts that interact in a non-simple way." Complex systems adapt or react to patterns created by multiple elements that constitute these systems[24]. Ludwig von Bertalanffy[25] wrote an influential paper generally accredited with bringing the 'systems perspective' into science, as an improvement or supplement to reductionist thinking. Systems/holistic thinking (especially in science) aims to connect patterns and contextualize the complexity as it is identified. The systems perspective must take off when reductionist thinking gets stuck. Ryan[26] chronicles the development of "systems approaches" and the "systems movement" starting with the introduction of von Bertalanffy's General Systems Theory (GST) in the midtwentieth century, the development of cybernetics, nonlinear dynamic systems, synergetics, complexity science and systems engineering.

The Walters Foundation[27] describes the following "Habits of Systems Thinkers":

1. Seek to understand the big picture; 2.Observe how elements within the system change over time, generating patterns and trends; 3. Recognize that a systems' structure (elements and interactions) generates behaviour; 4. Identify the circular nature of complex cause-and-effect relationships; 5. Surface and test assumptions; 6 . Change perspective to increase understanding; 7 . Consider an issue fully and resist the urge to come to a quick conclusion; 8. Consider how mental models affect current reality and the future; 9. Use understanding of system structure to identify possible leverage actions; 10. Find where unintended consequences emerge; 11. Recognize the impact of time delays when exploring cause-and-effect relationships; 12 . Check results and change actions if needed: "successive approximation."

Why is holistic/complexity/systems/nonlinear thinking superior? This perspective produces more insight for the manager, especially when she is presented with complex systems. Through this perspective, causality at the heart of patterns of change and adaptation in complex systems are revealed, especially those characteristics that have long-term effects. Senge[6] believes this approach assumes that there are underlying interrelationships at deeper levels in systems, and these interrelationships, once understood, will present a 
unique ability to influence change. Although these deeper levels in systems cannot be understood completely, plateaus of insight can be reached.

\section{Management issues: Holistic Perspective is superior}

\section{$3.1 \quad$ Business management}

For the opening paragraph in his seminal article in the Harvard Business Review, Forrester[28] wrote:

Management is on the verge of a major breakthrough in understanding how industrial company success depends on the interaction between the flows of information, materials, money, manpower, and capital equipment. The way these five flow systems interlock to amplify one another and to cause change and fluctuation will form a basis for anticipating the effects of decisions, policies, organizational forms, and investment choices.

That 'breakthrough' was the management methodology that came to be known as Systems Dynamics (SD). This application of the systems thinking/perspective has proved successful in business management for decades. SD as a field of research and analysis was founded at the Massachusetts Institute of Technology (MIT), by Jay Forester in the 1950s, for understanding the dynamic behaviour of problems and issues related to business enterprise. SD was first used by Forrester to solve a business problem for General Electric (GE). GE was disturbed by fluctuations in demand for the products from its household appliance plant. Forrester explained that five networks are key to understanding most industrial activity: materials, orders, money, capital equipment and personnel. There is a sixth network which connects these five networks, the information network[29]. Cause-and-effect is not usually linear in industrial (business) systems, it is difficult to measure because of the feedback delays in the information network. But, when links in the systems are understood, problems can be managed relatively easily. Usually a 'high leverage point' in the system is tweaked, usually a small yet consequential change in business practice. A landmark work in systems dynamics literature is the book Industrial Dynamics penned by Forrester[30]. Forrester showed that companies' internal policies were often the root-cause of most difficulties companies encounter, not competitors or market trends. So, instead of solving problems, company policies were often creating problems! 
When the SD program at MIT began under Forrester, he stated four goals of the program (then known as industrial dynamics):

1. To develop in the manager a better intuitive feel for the timevarying behaviour of industrial and economic systems. The study of particular situations....improves one's judgment about the factors influencing company success. The results are beneficial even before one feels that he has reached an accurate quantitative formulation of company behaviour that will have reliable predictive value.

2. To provide a background showing how the major aspects of a company are related to one another, so that the developing manager can derive the greatest benefit from his work experience. His operating experience should be more meaningful if he has a better understanding of how his immediate environment is related to the other company functions.

3. To help predict the future course of an existing organization. Very often present decisions determine future company welfare five years or more ahead...underlying, unchanging characteristics of a company can cause erratic manifestations in operations. We can hope, eventually, for better ability to see where present company practices will lead.

4. To improve the future prospects of a company. Beyond prediction lies the ability to redesign an organization and its policies so that it stands a better chance of success.

In Forrester's view, the manager's task is to understand the company as a system containing distinct but related separate functions, recognize that flows in this system influence how the market and the company relate and support each other. The manager must "interweave the tangible economic variables with the intangible variables of psychology and power structure"[31]. In SD, the mapping out of a company is not synonymous with the sketching out of an organizational chart. The dynamic system structure of the SD model consists of action streams, controlled by decision points and information flows. At any particular time in the system one person may be related to many decision points that control flow rates in different directions. So, the contributions from different persons or levels in the company may through one particular policy be represented at a decision stream.

This non-hierarchical interconnected systems view of a business enterprise has influenced the way many companies are now structured. GE's current CEO, Jeff Immelt described his "company's evolving culture that's focused on decentralized decision-making, speed and startup-like mentality"[32]. The 
most admired technology companies in the world like Microsoft, Google and Facebook are known for their flat structure that emphasize information flows and interrelationships. Slaughter[33] cited Google as the best example of a company where employees are encouraged to be innovative (and counter orthodoxies if necessary), this is only possible because the company is structured in an almost nonhierarchical manner.

The raison d'être of business strategy has always been thought of as competition of firms for market share. This is more of a reductionist perspective of 'strategy'. Thinking holistic, Arnold Hax, a professor of management at MIT sees complexity and thinks otherwise. Hax[34] believes that the relevant scope for business strategy is the 'extended enterprise', this is the network of the business firm, critical suppliers, customers and what he calls 'complementors.' Complementors are other firms (normally considered as competitors) that also deliver products and services in the industry, but these complementor firms also enhance delivery of the services and products by the firm in focus. A holistic/systems perspective views relationships with other firms as more symbiotic than competitive.

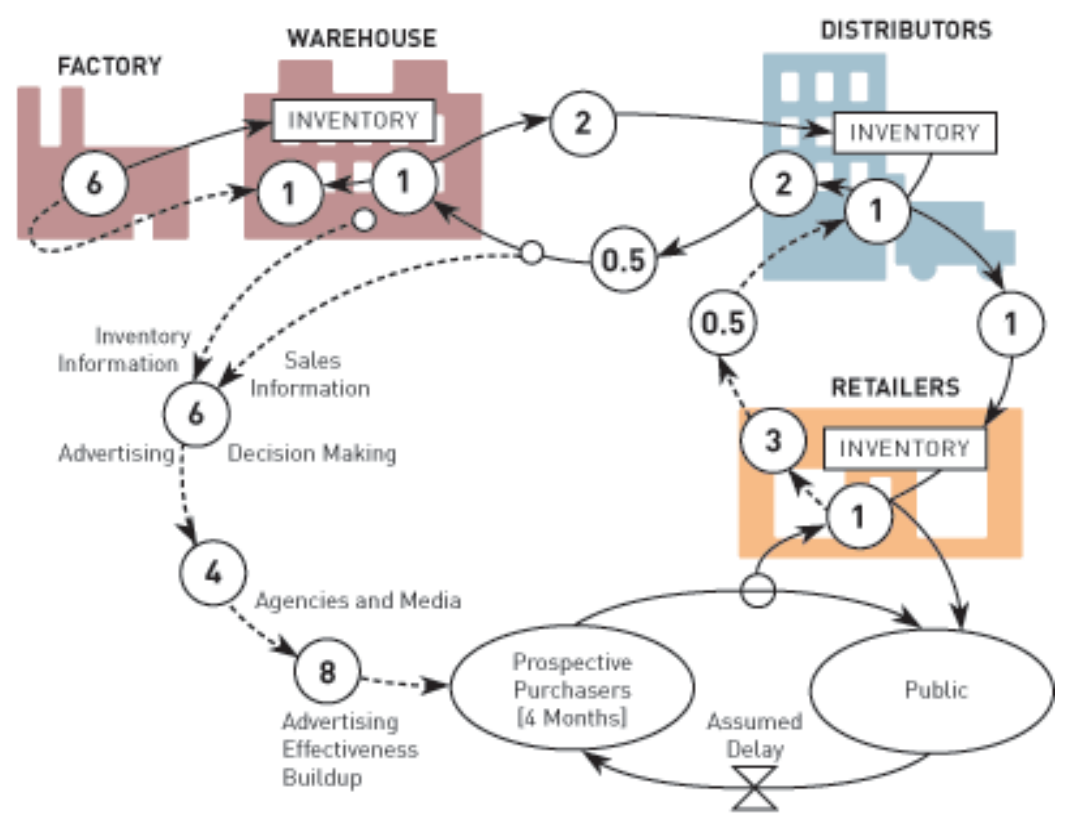

Figure 1: A systems dynamics model of GE's supply chain [28] 


\subsection{The economy}

The simple fact that the economy is a complex system is often ignored by professional economists. Arthur[24] was optimistic that after two centuries of economists studying static patterns with no behaviour adjustments (equilibria), economists had begun studying structures and unfolding patterns (emergence) in the economy. A holistic/systems/non-linear/complexity perspective sees the economy as an organic, constantly evolving unpredictable and non-deterministic system. Economic agents are constantly changing, adjusting: be they firms, banks, investors or consumers. Battiston et al.[35] suggest that economic policy must make use of behavioural modelling and network analysis, because of the complex nature of the economy. Traditional economic theory fell short of an explanation for the financial crisis of 2008, mainstream economists could not predict the crisis. Complexity theory has slowly but surely entered economic analysis. 'Contagion' 'networks' 'feedback' 'tipping points' are some of the concepts now used by economists borrowed from complexity/holistic/systems thinking. Because of the interrelatedness of agents in the economy, the global financial network of banks can fail even if individual banks appear unharmed. When existing economic modelling approaches fail, complexity/systems modelling must be developed.

Farmer and Foley[36] propose that the economy needs agent-based modelling, to replace the standard dynamic stochastic general equilibrium (DSGE) modelling favoured by economists in general. Agent-based modelling is superior to conventional equilibrium models: they cope better with non-linear behaviour (typical of complex systems), found in the economy. Agent-based models could model financial systems as the complex systems they really are. Forrester[37] created the Systems Dynamics National Model to "show how local policies governing decentralised decisions in an economy create observed overall economic behaviour." Much of prior work in economic modelling was declared (by Forrester) as inconsistent with a proper dynamic/systems/complexity perspective to economic behaviour. Forrester believed that his team had made progress in areas that had proved mysterious to most economists for a century. Forrester's model was able to endogenously generate economic growth, stagflation, business cycles, inflation, the economic long wave - major observed modes of behaviour in the economy.

It could be argued that the great economist F.A. Hayek[38], dealt a serious blow to the concept of central planning of economies, when he showed that 
the economy is a complex system, and a systems/holistic perspective is required to appreciate the role that information flows play. Hayek argued that central planning governments would never be able to manage the complexity of the economic system.

\subsection{Climate change}

Sterman[39] reveals the stark contrast between the overwhelming scientific consensus, about the reality and dangers of climate change, and the general public's complacency and sometimes confusion. The "foot-dragging", "waitand-see", "go-slow" attitude was displayed by 54 percent of interviewed people, when asked about combating climate change. The reason why half of the public believe that climate change risks can be tackled later, is due to a lack of holistic/systems/complexity thinking. The procrastination position would be tolerable if the climate system was a simple linear system, but it is not. According to Sterman "there are substantial delays in every link of a long causal chain stretching directly from the implementation of emissions abatement policies to emissions reductions to changes in atmospheric greenhouse gas concentrations to surface warming to changes in ice sheets, sea level, agricultural productivity, extinction rates, and other impacts." The climate system is a complex dynamic system with long delays, nonlinearities, feedbacks - not a simple linear system where the space between cause and effect is short and straight-forward. Hence, the wait-and-see approach is not sustainable.

\subsection{Global terrorism}

Combating global terrorism ranks very high in the priority list of most people. Yet, the knee-jerk reaction that most people and governments often advocate in fighting terrorism is short-sighted. This type of reductionist/linear thinking assumes simple cause-and-effect relationships in the fight against global terror. A holistic/systems/complexity perspective sees resistance against terrorism embedded in a network of complex cause-andeffect relationships with multiple feedback and non-linearity. A holistic approach will ensure that the disease of global terrorism is progressively eradicated, not just the symptoms. Propaganda may be just as effective as missiles for deterring terrorism. 'Winning the hearts and mind' of dispossessed, confused and vulnerable people in societies exposed to recruiters for terrorism is paramount. Intelligence gathering is critical: not just intelligence of where the next act of terrorism may be carried out, but intelligence about the lifestyles, backgrounds and networks of the criminals. 
Slaughter[33], describing the power that flows form a systems perspective of networks, states that the United States of America made progress in the fight against terrorism partly because of a dense global network of law enforcement officers, counterterrorism officials and intelligence agencies all over the world.

According to Searle[40]:

The human dimension of terrorism centres on, but is not restricted to, the individual. It operates also at the strategic level. For sure, those who set out to counter terrorism need to comprehend fully the big picture and to respond accordingly. Seismic political shifts will probably be necessary in the form of previously unconscionable changes of policy. An assured supply of data about terrorist organisations' structures, dispositions and intentions will be vital to stop attacks and apprehend those responsible. Big government initiatives may take place to generate counter-narratives. It's vital to have an appreciation of the terrorist organisation as a network of relationships and a human organism with intellectual and emotional depth.

Slaughter[5]calls for the constant creation of integrated networks of corporate, public and civic actors in response to the networks of bad actors that constantly threaten global security.

\subsection{Innovation/progress}

A recent newspaper headline startled the general public: it claimed that Charles Darwin was "no heroic genius" if a paper published in a scholarly journal is to be believed[41]. "Innovations from the likes of Darwin, Albert Einstein or Steve Jobs arise through societies and social networks acting as 'collective brains'." The article was based on a paper by Muthukrishna and Henrich[42]. The authors make use of a holistic/systems perspective of innovation and human progress, instead of seeing innovation and human progress as mostly the work of a talented few, whose achievements or products are then passed on to the general public. Muthukrishna and Henrich argue that cultural evolution is cumulative and the process of this evolution releases technologies and techniques. These technologies and techniques are not created by individuals on their own, in isolation, but in a milieu. More ideas and hence innovation and progress will likely be seen in larger more interconnected societies. Muthukrishna and Henrich pushed forward this startling hypothesis in their paper: 
Children are taught that Edison (or Swan) invented the light bulb, Gutenberg the printing press, Newton 'the calculus' and Ford the automobile. The underlying intuition is that innovation is an individual endeavour, driven by heroic geniuses and then passed on to the masses...We instead argue that innovations, large or small, do not require heroic geniuses any more than your thoughts hinge on a particular neuron. Rather, just as thoughts are an emergent property of neurons firing in our neural networks, innovations arise as an emergent consequence of our species' psychology applied within our societies and social networks. Our societies and social networks act as collective brains. Individuals connected in collective brains, selectively transmitting and learning information, often well outside their conscious awareness, can produce complex designs without the need for a designer-just as natural selection does in genetic evolution. The processes of cumulative cultural evolution result in technologies and techniques that no single individual could recreate in their lifetime, and do not require its beneficiaries to understand how and why they work. Such cultural adaptations appear functionally well designed to meet local problems, yet they lack a designer.

This insight has profound implications for society. The most innovative organizations and institutions seem to have put this insight to work. The interrelatedness of employees at Google or Microsoft, for example, is encouraged: the whole organization in these companies is structured to encourage cooperation and collaboration. Educational institutions that foster innovation like MIT are structured to encourage collaboration between students rather than competition between them. MIT does not grade its students' degrees in the traditional class designations: first class, second class or summa cum laude, magna cum laude etc., because this encourages competition between students, instead all MIT certificates contain the same class of degree (only GPAs defer).

\subsection{Ethnic violence/conflict/segregation}

To gain good undestanding of phenomena like ethnic violence, perpetual conflict and segeration, holistic thinking is needed. These are complex phenomena that usually have multiple underlying causes, or causes that are not always evident at first. Schelling[43] showed that separate decisions by individuals (individual choices) regarding choice of habitation, could lead to unorganized (untintentional) patterns of segregation: "a complex system with collective results that bear no close relation to individual intent" results. 
Lim et al.[44] further revealed that boundries between regions could be a source of violence if they are not well defined; especially when regions are differentiated by culture, through a pattern formation process (the process was identified by Lim et al.). Thinking from the holistic perspective sees such phenomena as containing complex processes of cause-and-effect, and policymakers informed by such scientific knowledge should be better equipped to prevent ethnic violence.

Through systems/complexity thinking, Lim et al. provide this key insight using spatial population structure:

\begin{abstract}
Violence arises due to the structure of boundaries between groups rather than as a result of inherent conflicts between groups themselves. In this [systems/complexity] approach, diverse social and economic causal factors trigger violence when the spatial population structure creates a propensity to conflict, so that spatial heterogeneity itself is predictive of local violence...ethnic violence can be studied in the universal context of collective dynamics, where models can identify how individual and collective behaviour are related.
\end{abstract}

\title{
3.7 Social policy
}

Social systems often behave counterintuitively. Social systems are complex dynamic systems, and causes of negative social effects can be far from the symptoms (temporarily and spatially). Social policy must take this nonlinear characteristic of social systems into consideration, when considering policy interventions. Policies intended to do good in the short-term may have longterm negative effects, and vice versa.

Forrester[45] takes a systems/complexty perspective to show that sometimes 'benevolent' policies produce unintended consequences. He explains that the policy to build low-income housing, can result in "depressed areas in cities" but this negative consequence is mistakenly assumed to be caused by housing shortage:

The legal and tax structures have combined to give incentives for keeping old buildings in place. As industrial buildings age, employment opportunities decline. As residential buildings age, they are used by lower-income groups who are forced to use them at higher population densities. Therefore, aging buildings cause jobs to decline and population to rise. Housing, at the higher population densities, accommodates more low-income 
urban population than can find jobs. A social trap is created where excess low-cost housing beckons low-income people inward because of the available housing...

\section{Conclusions}

The switch from reductionist to holistic thinking is imperative if managers will competently handle complex issues, in this world of complexity we live in. Reductionist thinking can be useful when dealing with more straightforward phenomena, with linear relationships and simple cause-and-effect, but such thinking often manifests as a blind-spot in managerial thinking. Scholars have shown -time and time again - the superiority of holistic thinking over reductionist thinking in various fields of human endeavour. In addition to such qualitative evidence presented in this paper, future research could seek for more quantitative measures that could highlight the loss to society, when the reductionist thinking mode is utilized with severe negative consequences, in contrast to the value that holistic / systems thinking would have produced in the same situation. An immediate example would be the monetary loss that resulted from the prevalence of reductionist thinking that contributed to the global financial crisis of 2008 .

\section{References}

[1] Petersen, E. and Plowman, G. (1946) Business Organization and Management (Revised Edition). Richard D. Irwin, Inc., New York.

[2] Sterman, J. (1994) Learning in and about Complex Systems. Systems Dynamics Review, 10, 291-330. https://doi:10.1002/sdr.4260100214

[3] Bar-Yam, Y. (1997) Complexity Rising: From Human Beings to Human Civilization, a Complexity Profile. NECSI Report 1997-12-01.

http://www.necsi.edu/EEA58B4C-0550-447D-8955

E3704D9223A1/FinalDownload/DownloadId-

E1201A8CD7337F9AEC1E41D698AAD872/EEA58B4C-0550-447D-8955E3704D9223A1/projects/yaneer/EOLSSComplexityRising.pdf

[4] Scharmer, O. (2010) Seven Acupuncture Points for Shifting Capitalism to Create a Regenerative Ecosystem Economy. Oxford Leadership Journal, 1, 
3: 1-21. https://www. presencing.com/resources/seven-acupuncturepoints-shifting-capitalism-creating-regenerative-ecosystem-economy

[5] Slaughter, A-M. (2016) How to Succeed in the Networked World. Foreign Affairs, November/December.

https://www. foreignaffairs.com/articles/world/2016-10-04/how-

succeed-networked-world

[6] Senge, P. (1990) The Fifth Discipline. Doubleday/Currency, New York.

[7] Forrester, J. (1971) Counterintuitive Behavior of Social Systems.

Technological Forecasting and Social Change, 3, 1-22.

https://doi.org/10.1016/S0040-1625(71)80001-X

[8] Cox, R. (2008) The Point is Not Just to Explain the World but to

Change It. Oxford Handbooks Online.

http://www.oxfordhandbooks.com/view/10.1093/oxfordhb/9780199219322.

$001.0001 /$ oxfordhb-9780199219322-e-4

[9] Mishra, P. (2016) Welcome to the Age of Anger. The Guardian.

https://www. theguardian.com/politics/2016/dec/08/welcome-age-angerbrexit-trump

[10] Adams, H (1918) The Education of Henry Adams. Houghton Mifflin, Boston, MA.

[11] Zuckerberg, M. (2017) Building Global Community.

https://web. facebook. com/notes/mark-zuckerberg/building-globalcommunity/10154544292806634/?_rdr

[12] The Sveriges Riksbank Prize in Economic Sciences in Memory of Alfred Nobel. (2002) Daniel Kahneman - Facts.

http://www.nobelprize.org/nobel_prizes/economic-

sciences/laureates/2002/kahneman-facts.html

[13] Kahneman, D. (2011) Thinking Fast and Slow. Farrar, Straus and

Giroux, New York.

[14] Robson, D. (2017) How East and West Think in Profoundly Different

Ways. http://www.bbc.com/future/story/20170118-how-east-and-west-

think-in-profoundly-different-ways

[15] Henrich, J., Heine, S. and Norenzayan, A. (2010) The Weirdest People in the World? Behavioural and Brain Sciences, 33, 61-135.

https://doi.org/10.1017/S0140525X0999152X

[16] Triandis, H. (1989) The Self and Social Behaviour in Differing Cultural Contexts. Psychological Review, 96 (3), 506-520.

https://doi.org/10.1037//0033-295x.96.3.506 
[17] Triandis, H. (1994) Culture and Social Behavior. McGraw-Hill, New York.

[18] Heine, S. (2007) and Hamamura, T. (2007) In Search of East Asian SelfEnhancement. Personality and Social Psychology Review, 1, 1-24.

https://doi.org/10.1177/1088868306294587

[19] Cross, P. (1977) Not Can but Will College Teaching be Improved. New Directions for Higher Education, 17, 1-15. https://doi.org/10.1002/he.36919771703

[20] Descartes, R. (1637) Discourse on Method and Related Writings, republished by Penguin Classics, London (2000). Penguin Classics, London.

[21] Wolfram, S. (2002) A New Kind of Science. Wolfram Media Inc, Champaign.

[22] Stanford Encyclopaedia of Philosophy (2002) Aristotle's Metaphysics. http://plato.stanford.edu/entries/aristotle-metaphysics/

[23] Simon, H. (1962) Proceedings of the American Philosophical Society, 106, 6, 467-482. http://www.jstor.org/stable/985254

[24] Arthur, W. (1999) Complexity and the Economy. Science, 284, 107-109. http://dx.doi.org/10.1126/science.284.5411.107

[25] von Bertalanffy, L. (1950) The Theory of Open Systems in Physics and Biology. Science, 111 (2872), 23-29.

https://doi.org/10.1126/science.111.2872.23

[26] Ryan, A. (2008) What is a Systems Approach?

https://arxiv.org/abs/0809.1698

[27] Waters Foundation (2017) Habits of a Systems Thinker. http://watersfoundation.org/systems-thinking/habits-of-a-systemsthinker/

[28] Forrester, J. (1958) Industrial Dynamics: A Major Breakthrough for Decision Makers. Harvard Business Review, July-August.

[29] Fisher, M. (2005) Prophet of Unintended Consequences. Strategy + Business, Issue 40, Autumn. https://www.strategybusiness.com/article/05308?gko=35c59

[30] Forrester, J. (1961) Industrial Dynamics. MIT Press, Cambridge MA.

[31] Forrester, J. (1968) Industrial Dynamics - After the First Decade.

Management Science, 14:7, 398-415.

http://dx.doi.org/10.1287/mnsc.14.7.398 
[32] Immelt, J (2016) Why GE is Giving up Employee Ratings, Abandoning Annual Reviews and Rethinking the Role of HQ.

https://www. linkedin.com/pulse/why-ge-giving-up-employee-ratingsabandoning-annual-reviews-

immelt?trk=vfeed\&lipi=urn\%3Ali\%3Apage\%3Ad_flagship3_feed\%3BSfAnqXWt hLTeQZ6Nv2UGHg\%3D\%3D

[33] Slaughter, A-M. (2009) America's Edge: Power in the Networked Century. Foreign Affairs, January/February.

https://www. foreignaffairs.com/articles/united-states/2009-0101/americas-edge

[34] Hax, A. (2010) The Delta Model: Reinventing Your Business Strategy. Springer-Verlag, New York.

[35] Battison, S., Farmer J., Flache, A., Garlaschelli, D., Haldane, A., Heesterbeck, H., Hommes, C., Jaeger, C., May, R. and Scheffer, M. (2016) Complexity Theory and Financial Regulation. Science, 351:6275, 818-819. https://doi.org/10.1126/science. aad0299

[36] Farmer, J. and Foley, D. The Economy Needs Agent-based Modelling. Nature, 460:6, 685-686. https://doi.org/10.1038/460685a

[37] Forrester, J. (1989) The System Dynamics National Model:

Macrobehaviour from Microstructure. Computer-Based Management of Complex Systems. Springer, Berlin, Heidelberg.

[38] Hayek, F. (1945) The Use of Knowledge in Society. The American Economic Review, 35:4, 520-530. http://www.jstor.org/stable/1809376

[39] Sterman, J. (2008) Risk Communication on Climate: Mental Models and Mass Balance. Science, 322, 532-533.

https://doi.org/10.1126/science.1162574

[40] Searle, N. (2017) To Defeat Terrorists We Have to Get into Their Minds. The Guardian.

https://www. theguardian.com/commentisfree/2017/mar/17/defeatterrorists-terrorism?CMP=share_btn_fb

[41] Morgan, T. (2016) Charles Darwin Was No 'Heroic Genius', Say Scientists. The Telegraph.

http://www.telegraph.co.uk/news/science/12176959/Charles-Darwinwas-no-heroic-genius-say-scientists.html

[42] Muthukrishna, M. and Henrich, J. (2016) Innovation in the Collective Brain, Philosophical Transactions of the Royal Society B, 371: 20150192. http://dx.doi.org/10.1098/rstb.2015.0192 
[43] Schelling, T. (1969) Models of Segregation. The American Economic Review, 59:2, 488-493. http://www.jstor.org/stable/1823701

[44] Lim, M., Metzier, R. and Bar-Yam, Y. (2007) Global Pattern Formation and Ethnic/Culural Violence. Science, 317, 1540-1544.

https://doi.org/10.1126/science.1142734 